\title{
Temporospatial expression and cellular localization of glutamine synthetase following traumatic spinal cord injury in adult rats
}

\author{
CHUNXING LIU ${ }^{1 *}$, WENJIE WU ${ }^{2 *}$, BIN ZHANG $^{3,4^{*}}$, JIE XIANG $^{4}$ and JIAN ZOU ${ }^{3,4}$ \\ ${ }^{1}$ Department of Clinical Testing, Huadong Sanitarium, Wuxi, Jiangsu 214065; ${ }^{2}$ Department of Pediatrics, \\ Shanghai Children's Medical Center Affiliated to Shanghai Jiaotong University School of Medicine, Shanghai 200127; \\ ${ }^{3}$ Department of Clinical Laboratory Science, Wuxi People's Hospital of Nanjing Medical University; \\ ${ }^{4}$ Wuxi Clinical Science Research Institute, Wuxi, Jiangsu 214023, P.R. China
}

Received October 30, 2012; Accepted March 13, 2013

DOI: $10.3892 / \mathrm{mmr} .2013 .1383$

\begin{abstract}
Glutamine synthetase (GS) is an enzyme involved in an endogenous mechanism of protection against glutamate neurotoxicity and is important in the regulation of astrocyte migration. To date, limited information is available concerning the expression of GS in normal spinal cords and following injury. In the present study, GS expression was identified in astrocytes, oligodendrocytes and microglia in normal rat spinal cords. Following traumatic spinal cord injury (SCI), the glutamate concentration increased rapidly at $1 \mathrm{~h}$ and returned to baseline rapidly. However, the GS activity and protein levels were found to decrease at $4 \mathrm{~h}$ and then increase gradually from day 3 following SCI. The quantification of astrocytes, oligodendrocytes and activated microglia/macrophages, as well as immunohistochemistry staining of day 7 post-injured spinal cords, indicated that the astrocytes and microglia/macrophages contributed to the increase in GS. Collectively, the results provided evidence for the temporospatial expression and location of GS following SCI and suggested that the changes in GS levels may contribute to glutamate neurotoxicity and glial cell response following $\mathrm{SCI}$.
\end{abstract}

\section{Introduction}

Spinal cord injury (SCI) is a devastating neurological injury that is associated with functional deficits and is a major cause of disability. The initial tissue damage in SCI is limited to the injury site. However, the damaged area gradually expands over time. One of the primary causes of secondary damage is

Correspondence to: Dr Jian Zou, Department of Clinical Laboratory Science, Wuxi People's Hospital of Nanjing Medical University, 299 Qingyang Road, Wuxi 214023, P.R. China

E-mail: zoujan@gmail.com

*Contributed equally

Key words: glutamine synthetase, spinal cord injury, glutamate, glial cells toxicity caused by excessive excitatory amino acids released from the injured tissue (1). Astrocytes are involved in the reduction of excitotoxicity through the conversion of glutamate into glutamine via glutamine synthetase (GS) (2-4). However, it is not clear whether the dysfunction of GS contributes to excitotoxicity-induced neuronal loss in SCI.

The activation of astrocytes leads to the formation of a glial scar, which inhibits axon regeneration and the maturation of neuronal tissues (5). Our previous study found that GS negatively regulates astrocyte migration and glial scar formation in $\mathrm{SCI}$, a process that is mediated by integrin $\beta 1$ and the matrix metalloproteinase, MT1-MMP (6). Thus, GS is a potential target for the regulation of astrocyte activation.

To date, however, only a limited number of studies have investigated the changes in GS levels following SCI (7-9). It is unknown whether these alterations in the GS levels contribute to neuronal loss and are associated with glial activation following SCI. In the present study, these issues were addressed by examining the temporospatial cellular expression of GS following SCI using a clinically relevant rat SCI model.

\section{Materials and methods}

Rat contusion injury and tissue preparation. Female Sprague-Dawley rats (200-220 g) were used for the induction of SCI. All animal experiments were approved by the Institutional Animal Care and Use Committee of Nanjing Medical University for the use of laboratory animals and performed in accordance with the Guide for the Care and Use of Laboratory Animals (National Research Council, 1996, USA). The rats were randomly assigned to control and experimental groups. Contusive SCI was performed as described previously $(6,10,11)$. Briefly, the rats were anesthetized by pentobarbital sodium $(50 \mathrm{mg} / \mathrm{kg}$; intraperitoneal injection) and the spinal cords were exposed by laminectomy at the thoracic 9 (T9) level. Body temperature was maintained at $37^{\circ} \mathrm{C}$ during anesthesia. Following the exposure of the dorsal surface of the spinal cord, with the dura remaining intact, a weight-drop injury was performed by dropping a 10 -g rod (diameter, $2.5 \mathrm{~mm}$ ) from a height of $25 \mathrm{~mm}$ using the New York University impactor (purchased from New York University Neurosurgery Laboratory, New York, NY, USA). 
Sham-operated rats were laminectomized but received no contusion. The rats were perfused transcardially with $100 \mathrm{ml}$ $0.9 \%$ saline followed by $200 \mathrm{ml} 4 \%$ paraformaldehyde at day 1 or week 1 post-injury. Following perfusion, the entire spinal cord was removed. The spinal cord tissue (cervical to sacral) was separated into 5-mm segments. Frozen transverse sections (20- $\mu \mathrm{m}$ thick) were obtained using a cryostat (Leica CM1900; Leica Microsystems, Inc., Nussloch, Germany) and thaw-mounted on poly-L-lysine-coated slides (Sigma, St. Louis, MO, USA). The results are representative of sections from 3 male and 3 female rats.

Immunofluorescence double-labeling. The immunofluorescence double-labeling method was performed as described previously (12). Briefly, the sections were premobilized and blocked with $0.3 \%$ Triton X-100 or $3 \%$ normal goat serum in $0.01 \mathrm{M}$ PBS for $30 \mathrm{~min}$ at room temperature (RT). A mixture of GS rabbit polyclonal (1:2,000; Abcam, Cambridge, UK) and mouse monoclonal antibodies were applied to the sections overnight at $4^{\circ} \mathrm{C}$. The mouse monoclonal antibodies used in this experiment included anti- $\beta$-III-tubulin (1:800; Sigma) to identify neurons, anti-glial fibrillary acidic protein (GFAP; 1:800; Sigma) to identify astrocytes, anti-RIP (1:25, a gift from Dr Scott R. Whittemore, University of Louisville, KY, USA) to identify oligodendrocytes, anti-OX42 (CD11b; 1:200; Chemicon, Temecula, CA, USA) to recognize resting microglial cells and anti-CD68 (1:200; R\&D systems, Minneapolis, MN, USA) to recognize activated microglia and macrophages. On the following day, the sections were incubated with FITC-conjugated goat anti-rabbit and TRITC-conjugated goat anti-mouse (1:100; Jackson ImmunoResearch Laboratories, Inc., West Grove, PA, USA) antibodies. The sections were washed, mounted and examined using the Olympus BX60 light (Olympus, Center Valley, PA, USA) or Zeiss LSM 510 confocal microscopes (Carl Zeiss AG, Oberkochen, Germany). Primary antiserum omission and normal mouse and goat serum controls were used to confirm the specificity of the immunofluorescent labeling.

Immunohistochemistry staining. Immunohistochemistry was performed using the avidin-biotinylated peroxidase complex $(\mathrm{ABC})$ immunoperoxidase-diaminobenzidine tetrahydrochloride (DAB) method according to the manufacturer's instructions (Zymed; Invitrogen Life Technologies, Carlsbad, CA, USA). Briefly, the sections were incubated with mouse anti-GS antibodies (1:1,000; BD Biosciences, Franklin Lakes, NJ, USA) and then reacted with biotinylated goat anti-mouse IgG for $0.5 \mathrm{~h}$ at RT and subsequently with $\mathrm{ABC}$ for $0.5 \mathrm{~h}$ at $\mathrm{RT}$. The reaction product was revealed by incubating the slices with DAB and $\mathrm{H}_{2} \mathrm{O}_{2}$ in substrate buffer for $15 \mathrm{~min}$. Next, the sections were dehydrated, cleared and coverslipped. The slides were examined using an Olympus BX60 light microscope. The primary anti-serum omission and normal goat controls were used to confirm the specificity of the immunohistochemical labeling.

Western blot analysis. Western blot analysis was performed as described previously (6). Briefly, a 10-mm cord segment containing the injury epicenter was removed and homogenized by sonication in a lysis buffer. Protein $(20 \mu \mathrm{g})$ from the sample supernatant was measured using the BCA method and loaded onto a $12 \%$ polyacrylamide gel, separated by SDS-PAGE and transferred to PVDF membranes by electrophoresis. Following membrane blocking, the mouse anti-GS (1:5,000; BD Biosciences) or anti-GFAP antibodies (1:1,000; Sigma-Aldrich) were added to the membrane and incubated at $4^{\circ} \mathrm{C}$ overnight. The membrane was washed and incubated with secondary antibody goat anti-mouse IgG conjugated with horseradish peroxidase (1:2,000; Amersham Pharmacia Biotech, Arlington Heights, IL, USA) at RT for $2 \mathrm{~h}$. Subsequent to being washed, the membrane was analyzed by enhanced chemiluminescence (Amersham Pharmacia Biotech).

GS activity measurement. GS activity in the tissue was measured using the colorimetric assay as described previously (3) and then expressed as c-glutamylhydroxamate $(\mu \mathrm{M}) / \mathrm{h} / \mathrm{mg}$ cell protein. The spinal cord $(10-\mathrm{mm}$ section containing the injury epicenter) was obtained at varying post-operative survival times. The tissue protein concentrations were determined using the BCA protein estimation kit (Pierce Biotechnology, Inc., Rockland, IL, USA).

Glutamate concentration analysis. The glutamate content in the spinal cord samples was quantified by measuring the absorbance at $492 \mathrm{~nm}$ using the Glutamate Colorimetric Assay kit (Genmed Scientific, Inc., Arlington, MA, USA) and expressed as glutamate $(\mu \mathrm{M}) / \mathrm{mg}$ tissue protein. A standard curve was constructed in each assay using known concentrations of glutamate. The concentration of tissue glutamate in the spinal cord (10-mm section containing the injury epicenter) was estimated from the standard curve.

Statistical analysis. All data are expressed as mean \pm SD. A one-way ANOVA with Tukey's HSD post hoc t-test was used to determine the level of statistical significance. $\mathrm{P}<0.05$ was considered to indicate a statistically significant difference.

\section{Results}

Distribution of GS in the rat spinal cord. Immunofluorescence double staining was employed to confirm the cellular localization of GS in the rat spinal cords. Sections were obtained from an area $5 \mathrm{~mm}$ caudal to the epicenter of the sham-operated rat spinal cord. GS-expressing glial cells, including GFAP-positive astrocytes (Fig. 1A-C), RIP-positive oligodendrocytes (Fig. 1D-F) and OX42-positive microglia (Fig. 1G-I) were identified. However, GS expression was not observed in $\beta$-III-tubulin-positive neurons (Fig. 1J-L).

Glutamine synthetase activity and protein levels following $S C I$. GS is the key glial-specificity enzyme that converts glutamate to glutamine to affect the clearance of synaptic glutamate (13). In the present study, the correlation between GS activity and glutamate concentration following SCI was analyzed. As shown in Fig. 2A, the concentration of glutamate increased rapidly and reached a maximum of $1.26 \pm 0.08$ fold at $1 \mathrm{~h}$ compared with the sham-operated group $(\mathrm{P}=0.0048)$ post-injury. The concentration returned to baseline rapidly at $4 \mathrm{~h}$ post-injury. Fig. 2B demonstrates that a significant decrease in GS activity occurred at $4 \mathrm{~h}$ post-injury $(\mathrm{P}=0.018)$, then the 

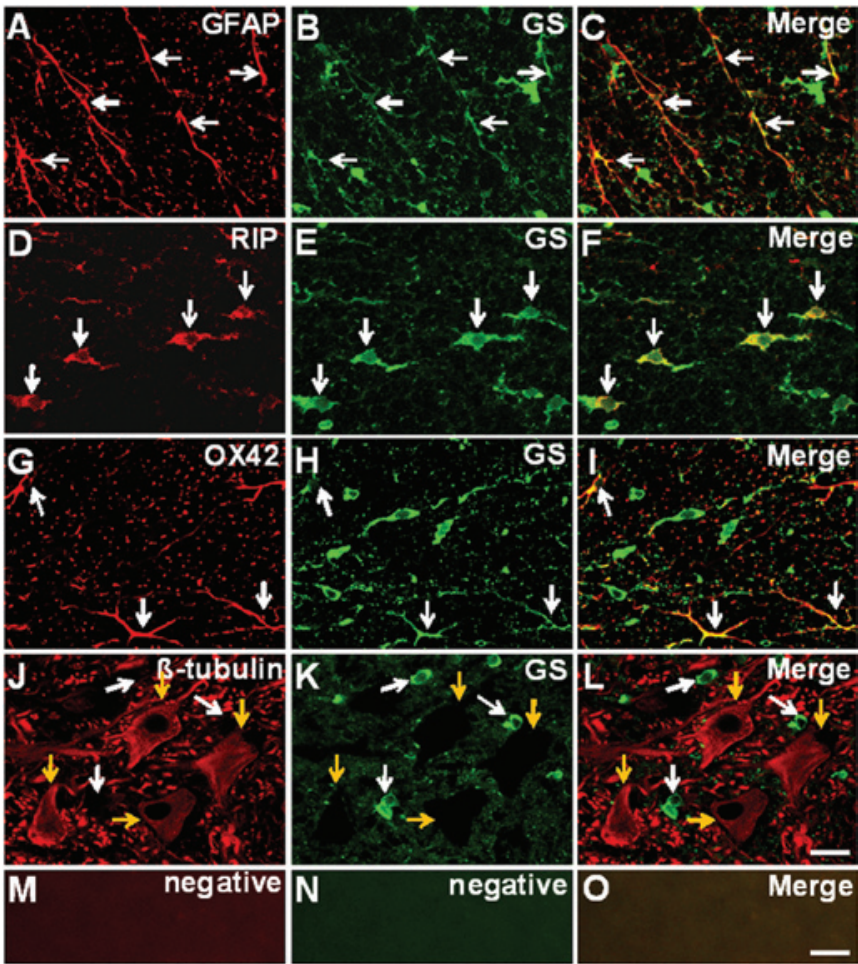

Figure 1. Cellular localization of GS in normal rat spinal cords by immunofluorescence double labeling. (A-C) GS colocalized in astrocytes (GFAP), (D-F) oligodendroctes (RIP) and (G-I) microglia (OX42). (A-I) White arrows indicate colocalization of GS with specific markers for each cell type. (J-L) Yellow arrows indicate that $\beta$-III-tubulin-positive neurons were negative for GS. (M-O) negative control. Scale bars, $20 \mu \mathrm{m}$. GS, glutamine synthetase; GFAP, glial fibrillary acidic protein.

GS activity increased, prior to returning to the basal level at day 1 and then increasing to peak levels at day 7 post-injury $(\mathrm{P}=0.0037)$. The GS activity then remained at a high level. SCI also caused a significant decrease in the GS expression at $4 \mathrm{~h}(\mathrm{P}=0.018)$ and day $1(\mathrm{P}=0.0079)$ post-injury. The GS protein levels also revealed a similar time course change following SCI (Fig. 2C and E). To test whether the change in GS levels following SCI was associated with the activity of glial cells, the correlation between the changes in GS and GFAP, CD68 and PLP, the specific markers for astrocytes, active microglia/macrophages and oligodendrocytes, respectively, was determined (Fig. 2C-H). The western blot analysis revealed that GFAP levels decreased $1 \mathrm{~h}$ post-injury, decreased further at $4 \mathrm{~h}(\mathrm{p}=0.018)$ and day $1(\mathrm{P}=0.0022)$ post-injury, then increased rapidly from day $3(\mathrm{P}=0.0099)$ and peaked at day 7 post-injury $(\mathrm{P}=0.000016)$. The GFAP protein concentration remained at a high level from then onwards (Fig. 2C and D). Fig. 2E and G revealed that CD68 was almost undetectable in the sham-operated spinal cords and increased significantly from $4 \mathrm{~h}$ following SCI ( $\mathrm{P}=0.0011$; Fig. $2 \mathrm{~F}$ and $\mathrm{G})$. However, the PLP expression decreased gradually and was significantly decreased 1 day after $\mathrm{SCI}(\mathrm{P}=0.0033$; Fig. $2 \mathrm{~F}$ and $\mathrm{H})$.

Distribution of GS in the injured spinal cords. To determine the spatial distribution of GS following SCI, immunochemistry staining for GS was performed at days 1 and 7 post-injury, when the GS protein was minimally and maximally expressed, respectively. In the sham-operated rats (Fig. 3, left column),
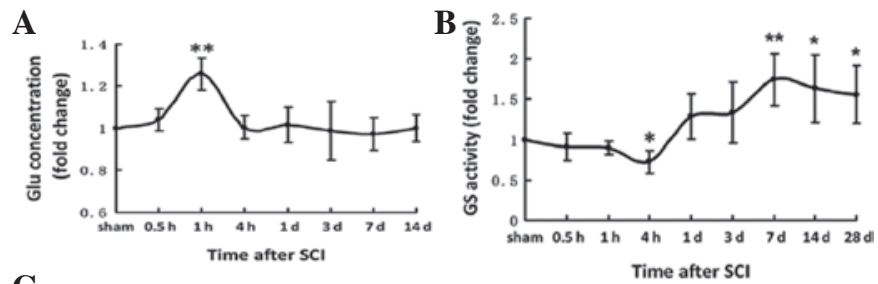

C

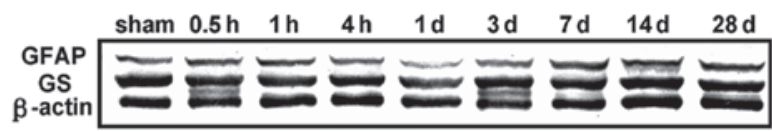

D

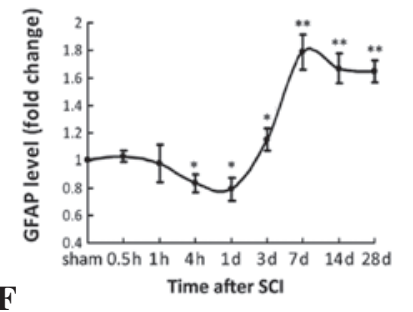

$\mathbf{E}$
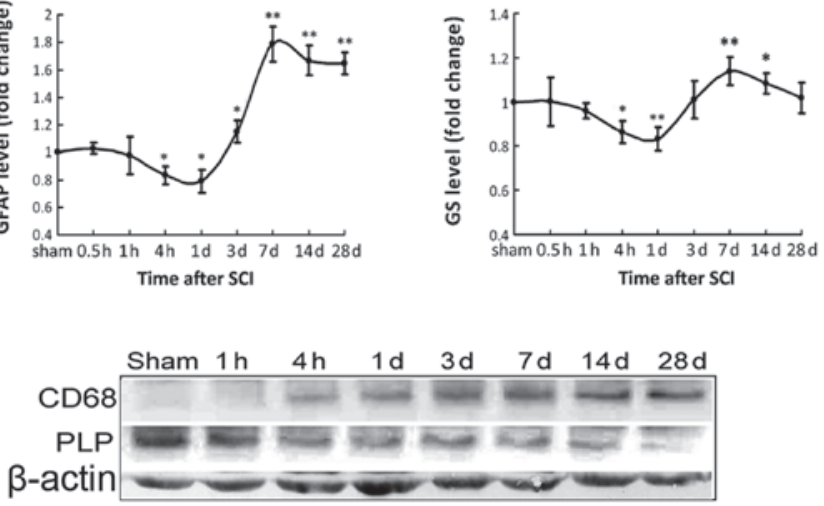

G

H
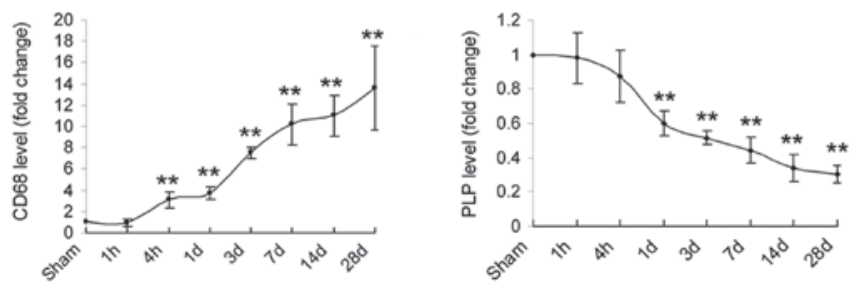

Figure 2. Glutamate concentration and GS activity changes following rat spinal cord injury (SCI). (A) Glutamate concentration and (B) GS activity changes in spinal cord at various times post-injury. (C) Representative bands of GFAP and GS. $\beta$-actin (42 kDa) served as a loading control. (D-E) Densitometrical analysis of (D) GFAP and (E) GS immunoreactive signals normalized against $\beta$-actin. (F-H) Representative bands of CD68 and PLP. (G-H) Densitometrical analysis of the (G) CD68 and (H) PLP immunoreactive signals normalized against $\beta$-actin. Data were normalized against the sham and are presented as mean $\pm \mathrm{SD}\left(\mathrm{n}=6 ;{ }^{*} \mathrm{P}<0.05\right.$ and ${ }^{* *} \mathrm{P}<0.01$, vs sham). GS, glutamine synthetase; GFAP, glial fibrillary acidic protein.

the GS immunoreactivities revealed comparable basal levels in the glial cells in the white and gray matter of the spinal cord. At day 1 post-injury, the GS immunoreactivities were markedly decreased in the injured epicenter dorsal column and ventral horn gray and white matter (Fig. 3B and E). However, no significant difference was observed in the GS levels in the region 5-mm caudal to the injury site (Fig. $3 \mathrm{H}$ and $\mathrm{K}$ ). By contrast, the GS immunoreactivities were markedly increased in the glial cells in the white and gray matter 7 days after SCI (Fig. 3C and F). The strongest labeling was observed at the lesion cavity and surrounding area. Specific GS immunoreactivities were morphologically characteristic of activated astrocytes. It appeared that certain cells with spherical morphologies were derived from cells that infiltrated into the lesion site. Furthermore, the increased GS immunore- 


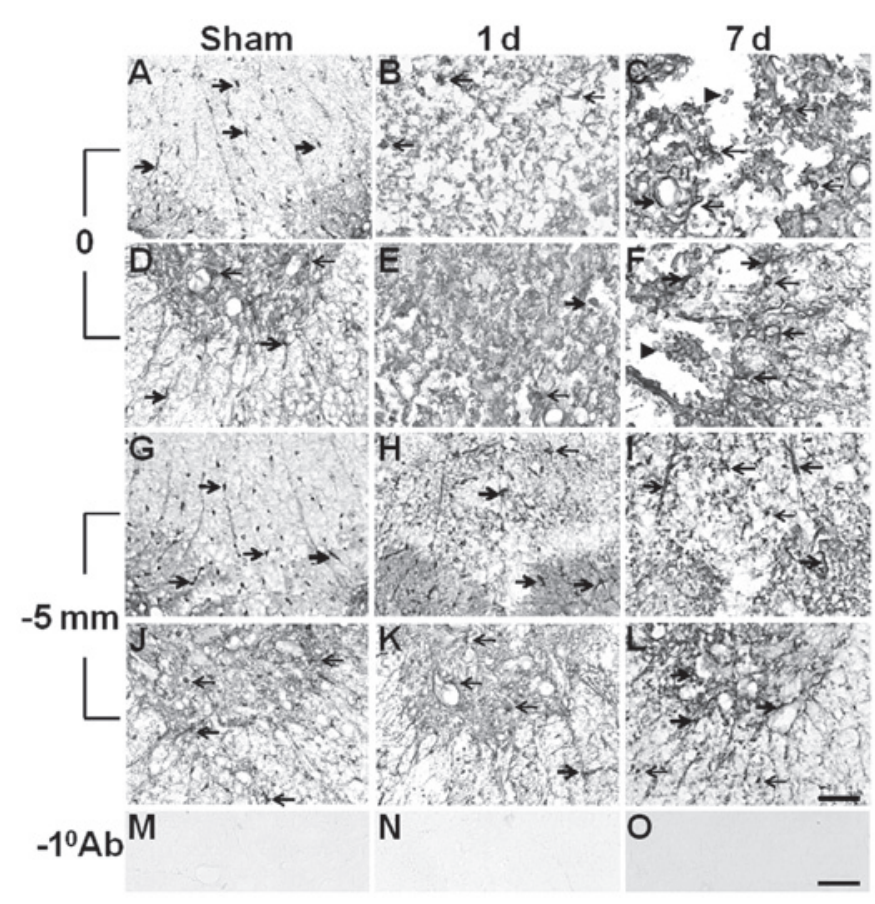

Figure 3. Distribution of GS immunoreactivites in the spinal cord of sham-operated and injured rats. (A, D, G and J) GS immunoreactivites were found in the white and gray matter glial cells in sham-operated spinal cords. (B,E,H and K) A marked decrease in GS was found in the injury epicenter at day 1 post-injury. No significant changes in GS were found in the regions 5-mm caudal to the injury site $(-5 \mathrm{~mm}$; $\mathrm{H}$ and $\mathrm{K})$. (C, F, I and L) A marked increase in GS was found in the (C and F) injury epicenter $(0)$ and ( $\mathrm{I}$ and $\mathrm{L}$ ) regions $5 \mathrm{~mm}$ caudal to the injury epicenter $(-5 \mathrm{~mm})$. (C and F) At the injury epicenter, numerous labeled cells were observed to be derived from cells that infiltrated into the lesion sites. (M-O) Controls without primary antibodies. Arrows indicate typical GS-positive cells. Scale bars, $50 \mu \mathrm{m}$. GS, glutamine synthetase.

activities were also observed throughout the entire length of the specimen (10 mm; Fig. 3I and L).

Cellular localization of GS in the injured spinal cord. The astrocyte response to injury was confined to the immediate penumbra surrounding the lesion center. Astrocyte reactivity was characterized by a cellular hypertrophy with a process extension and increased production of intermediate filaments, including GFAP (Fig. 4A). The upregulation of GS in the tissue surrounding the lesion center was produced by GFAP-positive astrocytes (Fig. 4A-D). Since macrophages and microglial cells express GS $(14,15)$, CD68 was used to identify the GS-positive infiltrative cells. As revealed in Fig. 4E-H, colocalization of GS and CD68 was observed in certain spherical cells (indicated by arrows). Thus, these results indicate that the infiltrative macrophages/activated microglia in areas close to the injury site express GS.

\section{Discussion}

In the present study, changes in the GS levels in the normal and injured rat spinal cords were systematically analyzed. To the best of our knowledge, this is the first study to demonstrate the temporospatial expression pattern of GS and its cellular localization in the adult rat spinal cord following SCI.

GS is expressed in glial cells, including astrocytes, oligodendrocytes and microglia in the white and gray matter in
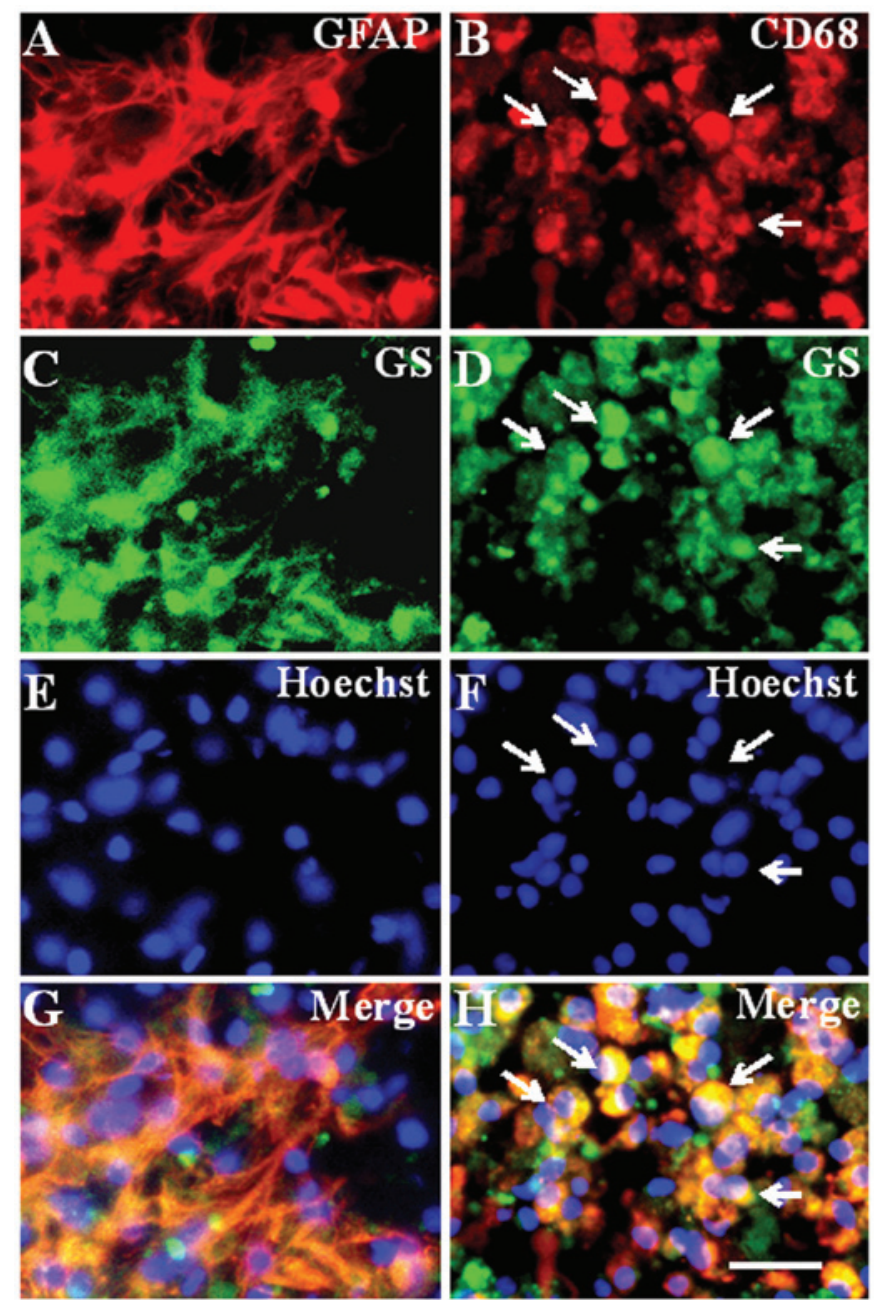

Figure 4. Increased GS immunoreactivity present in the epicenter of the spinal cord 7 days post-injury. Antibodies against (A) GFAP-labeled astrocytes, (B) CD68-labeled macrophages and (C) activated microglia. (C and G) Nuclei labeled with Hoechst. (G and H) Merged images. (A-D) GS was expressed in the astrocytes, with marked GFAP-immunoreactivity in the surrounding cavity. (E-H) CD68-immunoreactive cells were GS positive in the center of the cavity. Arrows indicate typical CD68-positive cells with GS staining. Scale bars, $20 \mu \mathrm{m}$. GS, glutamine synthetase; GFAP, glial fibrillary acidic protein.

normal rat spinal cords. The enzyme is associated with the regulation of glutamate concentration in the synaptic claft (2) and therefore the expression of GS in these supporting cells is vital to normal neural function $(13,16)$. Our recent study revealed that MMPs are regulated by GS in astrocytes (6). Since MMPs in the CNS have been implicated in synaptic plasticity, neuroprotection, oncogenesis and oligodendrocyte differentiation (17), the effects of GS in the regulation of neuronal function may be mediated by MMPs. GS may exert its effect on astrocytes and Schwann cell differentiation by regulating glutamate concentration $(2,18)$. These observations indicate that GS may play roles in the differentiation of oligodendrocytes and astrocytes. However, the function of GS in the microglia is not well documented.

GS is a key glutamate-metabolizing enzyme and the removal of extracellular glutamate is dependent on the expression level of this enzyme in glial cells $(4,19)$. In the present study, the activity and expression of GS were initially maintained at low 
levels following SCI, which was consistent with the marked elevation of glutamate at the injury site. The downregulation of GS and the increased levels of glutamate may be caused by the marked destruction of neuronal and glial cells, including astrocytes and oligodendrocytes, in the acute stage following SCI. On the other hand, the initial inflammation triggered by SCI is mediated by the cytokines and their receptors, including TNF- $\alpha$ and its receptor $(20,21)$. These cytokines induce neural excitotoxicity in glial cells (22) by suppressing GS expression in astrocytes (3) and may explain why an increase in active microglia/macrophages at the early stages subsequent to SCI did not induce an increase in GS, despite the cells expression of GS. Thus, according to our previous study, these results indicate that low levels of GS and a robust induction of cytokine expression at early stages following SCI induces cell death by excitotoxicity. However, the mechanism by which GS is downregulated during the initial activation of the glial cells is largely unknown.

Following CNS injury, astrocytes undergo a transformation from a quiescent to a reactive state, in a process known as dedifferentiation (23). Previous studies have demonstrated that GS is associated with gliosis following injury $(8,24)$ and that downregulation of GS is associated with the activation or dedifferentiation of astrocytes (25). However, the role of GS in astrocyte activation remains unclear. Our recent study demonstrated that the downregulation of GS promotes the migration of astrocytes, a process mediated by the upregulation of MT1-MMP and downregulation of integrin $\beta 1$ (6). The results suggested that the temporal downregulation of GS in astrocytes at the early stages of SCI is a promising strategy for SCI treatment, which induces astrocytic activation and migration in order to restrict the expansion of the inflammatory and lesional areas.

In the central nervous system, the importance of GS in glutamate metabolism has been largely studied with respect to excitotoxicity and/or glutamatergic neurotransmission between neurons and astrocytes (26). The present study revealed that GS is also expressed in oligodendrocytes in the spinal cord. However, the function of GS in these cells has not been clearly described. A previous study demonstrated that GS is not only a metabolically relevant enzyme, but that it also regulates Schwann cell differentiation and promotes myelination by regulating the glutamate concentration (18). These observations may aid further studies in revealing the functional significance of GS in oligodendrocytes and during the development and degeneration of the central nervous system.

In the present study, macrophages and activated microglia were found to invade the lesion sites 7 days post-injury and exhibit GS-positive immunoreactivities. These results indicate that, in addition to their recognized neurotoxic properties in inflammation following SCI, these cells may exhibit specific neuroprotective properties, which partly compensate for the inhibition of astrocytic function. By contrast, microglia express glutaminase, the enzyme which catalyzes the conversion of glutamate from glutamine. A previous study reported that TNF- $\alpha$ induced neurotoxicity via glutamate release from activated microglia (27). Therefore, the function of GS in these cells is complex. The clarification of the correlation between GS and inflammation or inflammatory cells must be performed as the function of GS in these cells is likely to form the basis for any future clinical applications.
In conclusion, the present study demonstrated that low levels of GS contribute to glutamate neurotoxicity at an early stage following SCI and that the activation of glial cells leads to changes in the GS levels. Understanding the function and mechanism of GS is a novel strategy for studying the role of astrocytes and microglia in the recovery of damaged tissue in SCI.

\section{Acknowledgements}

The current study was supported by grants from the Natural Science Foundation of China (no. 81000527), the Natural Science Foundation of Jiangsu Province (no. BK2010159) and the Science and Technology Development Program of Huadong Sanitarium (no. 201001). The authors would like to thank BiomedWorld Bioscience Ltd., Co., for their editing of the manuscript.

\section{References}

1. Mills CD, Xu GY, McAdoo DJ and Hulsebosch CE: Involvement of metabotropic glutamate receptors in excitatory amino acid and GABA release following spinal cord injury in rat. J Neurochem 79: 835-848, 2001.

2. Suárez I, Bodega G and Fernández B: Glutamine synthetase in brain: effect of ammonia. Neurochem Int 41: 123-142, 2002.

3. Zou J, Wang YX, Dou FF, et al: Glutamine synthetase down-regulation reduces astrocyte protection against glutamate excitotoxicity to neurons. Neurochem Int 56: 577-584, 2010.

4. Xu B, Xu ZF, Deng Y, Liu W, Yang HB and Wei YG: Protective effects of MK-801 on methylmercury-induced neuronal injury in rat cerebral cortex: involvement of oxidative stress and glutamate metabolism dysfunction. Toxicology 300: 112-120, 2012.

5. Silver J and Miller JH: Regeneration beyond the glial scar. Nat Rev Neurosci 5: 146-156, 2004.

6. Zou J, Wang YX, Mu HJ, et al: Down-regulation of glutamine synthetase enhances migration of rat astrocytes after in vitro injury. Neurochem Int 58: 404-413, 2011.

7. Baldwin SA, Broderick R, Blades DA and Scheff SW: Alterations in temporal/spatial distribution of GFAP- and vimentin-positive astrocytes after spinal cord contusion with the New York University spinal cord injury device. J Neurotrauma 15: 1015-1026, 1998.

8. Benton RL, Ross CD and Miller KE: Glutamine synthetase activities in spinal white and gray matter 7 days following spinal cord injury in rats. Neurosci Lett 291: 1-4, 2000.

9. Romero-Alemán MM, Monzón-Mayor M, Yanes C and Lang D: Radial glial cells, proliferating periventricular cells, and microglia might contribute to successful structural repair in the cerebral cortex of the lizard Gallotia galloti. Exp Neurol 188: 74-85, 2004.

10. Lu HZ, Wang YX, Zou J, et al: Differentiation of neural precursor cell-derived oligodendrocyte progenitor cells following transplantation into normal and injured spinal cords. Differentiation 80: 228-240, 2010.

11. Lü HZ, Xu L, Zou J, et al: Effects of autoimmunity on recovery of function in adult rats following spinal cord injury. Brain Behav Immun 22: 1217-1230, 2008.

12. Hu JG, Fu SL, Zhang KH, et al: Differential gene expression in neural stem cells and oligodendrocyte precursor cells: a cDNA microarray analysis. J Neurosci Res 78: 637-646, 2004.

13. Eid T, Behar K, Dhaher R, Bumanglag AV and Lee TS: Roles of glutamine synthetase inhibition in epilepsy. Neurochem Res 37: 2339-2350, 2012.

14. Chrétien F, Vallat-Decouvelaere AV, Bossuet C, et al: Expression of excitatory amino acid transporter-2 (EAAT-2) and glutamine synthetase (GS) in brain macrophages and microglia of SIVmac251-infected macaques. Neuropathol Appl Neurobiol 28: 410-417, 2002.

15. Caldani M, Rolland B, Fages C and Tardy M: Glutamine synthetase activity during mouse brain development. Experientia 38: 1199-1202, 1982.

16. Coulter DA and Eid T: Astrocytic regulation of glutamate homeostasis in epilepsy. Glia 60: 1215-1226, 2012. 
17. Moore CS and Crocker SJ: An alternate perspective on the roles of TIMPs and MMPs in pathology. Am J Pathol 180: 12-16, 2012.

18. Saitoh F and Araki T: Proteasomal degradation of glutamine synthetase regulates schwann cell differentiation. J Neurosci 30 : 1204-1212, 2010.

19. Shaked I, Ben-Dror I and Vardimon L: Glutamine synthetase enhances the clearance of extracellular glutamate by the neural retina. J Neurochem 83: 574-580, 2002.

20. Yan P, Liu N, Kim GM, et al: Expression of the type 1 and type 2 receptors for tumor necrosis factor after traumatic spinal cord injury in adult rats. Exp Neurol 183: 286-297, 2003.

21. Wang XF, Huang LD, Yu PP, et al: Upregulation of type I interleukin-1 receptor after traumatic spinal cord injury in adult rats. Acta Neuropathol 111: 220-228, 2006.

22. Xie Z, Smith CJ and Van Eldik LJ: Activated glia induce neuron death via MAP kinase signaling pathways involving JNK and p38. Glia 45: 170-179, 2004.
23. Lang B, Liu HL, Liu R, Feng GD, Jiao XY and Ju G: Astrocytes in injured adult rat spinal cord may acquire the potential of neural stem cells. Neuroscience 128: 775-783, 2004.

24. Politis MJ and Miller JE: Post-traumatic alterations in glutamine synthetase activity in peripheral and central nerves. Brain Res 359: 183-186, 1985.

25. Egnaczyk GF, Pomonis JD, Schmidt JA, et al: Proteomic analysis of the reactive phenotype of astrocytes following endothelin-1 exposure. Proteomics 3: 689-698, 2003.

26. Levy HL: Metabolic disorders in the center of genetic medicine. N Engl J Med 353: 1968-1970, 2005.

27. Takeuchi H, Jin S, Wang J, et al: Tumor necrosis factor-alpha induces neurotoxicity via glutamate release from hemichannels of activated microglia in an autocrine manner. J Biol Chem 281: 21362-21368, 2006. 